\title{
Estudio del mecanismo de reacción de la oxidación de isopropanol sobre óxidos mixtos de Mn-Ti mediante espectroscopía FT-IR y cromatografía de gases
}

\author{
Lab. de Altas Presiones. Dpto. Q. Inorgánica I, Universidad Complutense, Ciudad Universitaria, E-28040 Madrid. \\ Istituto di Chimica, Università di Genova, P.le J.F. Kennedy, I-16129 Génova (Italia). \\ Dpto. Q. Inorgánica, Universidad Salamanca, Pa Merced, E-37008 Salamanca.
}

\begin{abstract}
Se han sintetizado varias muestras de óxido de manganeso soportado sobre óxido de titanio anatasa mediante un método de impregnación húmeda convencional, denotándolos con la fórmula general $\mathrm{XMn}-\mathrm{TiO}_{2}$ (con $\mathrm{X}=0.5,1.0,1.5$ y 2.0, fracciones teóricas de la monocapa para cubrir el soporte). Todas las muestras se caracterizaron con las técnicas convencionales para el estudio del estado sólido. Se encontró que el óxido de manganeso se encuentra bien dispersado sobre la superficie del soporte en forma de especies $\mathrm{Mn}^{3+}$, mientras que otra parte se estabiliza en el interior de la estructura del TiO $\mathrm{T}_{2}$. Estos materiales son estables en el intervalo de temperaturas $600-900 \mathrm{~K}$ y presentan superficies específicas medias o altas entorno a un valor medio de $80 \mathrm{~m}^{2} / \mathrm{g}$. Desde el punto de vista catalítico, los óxidos mixtos de $\mathrm{Mn}$ y Ti son menos reactivos que los óxidos de manganeso, $\mathrm{Mn}_{2} \mathrm{O}_{3}$ y $\mathrm{Mn}_{3} \mathrm{O}_{4}$, en la oxidación de isopropanol, aunque sobre $600 \mathrm{~K}$ se alcanza la combustión total de isopropanol a $\mathrm{CO}_{2}$ y dependiendo de la composición de Mn llegan a ser más selectivos a propileno. El estudio conjunto mediante espectroscopía FT-IR y cromatografía de gas-masas complementa los resultados de las pruebas catalíticas permitiendo detectar intermedios inestables de reacción, como formiatos y ésteres, y así proponer un camino de reacción en la superficie del catalizador formado por 2-propóxido/acetato/formiato.
\end{abstract}

Palabras Clave: espectroscopía FT-IR, óxido de manganeso, formiato, isopropanol, propeno, oxidación

A reaction mecanism study of isopropanol oxidation on Mn-Ti mixed oxides by FT-IR spectroscopy and mass-gas cromatograph

Several samples of $\mathrm{TiO}_{2}$ (anatase)-supported Mn oxide have been prepared by a wet impregnation method, named as XMn$\mathrm{TiO}_{2}$ (with $\mathrm{X}=0.5,1.0,1.5$ and 2.0, the theoretical monolayer fractions). All samples were characterised by conventional techniques. Mn oxide was found to be well dispersed on the support surface as $\mathrm{Mn}^{3+}$ species, while part of it is stabilised into the $\mathrm{TiO}_{2}$ bulk as $\mathrm{Mn}^{4+}$ species. These materials are stable in the 600-900 $\mathrm{K}$ range and retain medium-high average specific surface area of $\sim 80 \mathrm{~m}^{2} / \mathrm{g}$. From the catalytic point of view, they are less active than pure $\mathrm{Mn}$ oxides, $\mathrm{Mn}_{2} \mathrm{O}_{3} \mathrm{y} \mathrm{Mn}_{3} \mathrm{O}_{4}$, in the isopropanol oxidation but, above $600 \mathrm{~K}$, reach complete oxidation of isopropanol to $\mathrm{CO}_{2}$ and depending on the Mn content become more selective to propilene. FT-IR studies and the GC-MS analysis confirm the catalytic test results allowing to detect some intermediate products, as formates and esthers, and so to propose the following 2-propoxide/acetate/formate surface pathway for the 2-propanol total oxidation.

Keywords: FT-IR spectroscopy, manganese oxide, formate, isopropanol, propilene, oxidation

\section{INTRODUCCIÓN}

Las emisiones gaseosas procedentes de la industria están formadas en su mayor parte por gases contaminantes del tipo $\mathrm{NO}_{x^{\prime}} \mathrm{SO}_{\mathrm{x}}$ y compuestos orgánicos volátiles (VOC). La eliminación puede realizarse mediante el proceso SCR para el $\mathrm{NO}_{x}$, en presencia de amoníaco y un catalizador cerámico (Pt- $\left.\mathrm{Al}_{2} \mathrm{O}_{3}, \mathrm{~V}-\mathrm{W}-\mathrm{Ti}\right)(1,2) \mathrm{y}$, en algunos casos, en presencia de los propios hidrocarburos $(3,4)$. En cambio el $\mathrm{SO}_{\mathrm{x}}$ tiene un problema adicional, pues su oxidación da lugar a sulfatos que envenenan el catalizador $(5,6)$. Así, en el caso de los motores diesel, se tiende a minimizar el azufre del propio combustible (7).

La tendencia actual en catálisis ambiental es la búsqueda de materiales con actividad catalítica que no sean contaminantes cuando se desactiven. En este sentido los óxidos de mangane- so $\left(\mathrm{Mn}_{3} \mathrm{O}_{4}(8), \mathrm{Mn}_{2} \mathrm{O}_{3}(9)\right.$ y $\left.\mathrm{MnO}_{2}(10)\right)$ están siendo objetivos de investigación por su conocida actividad en la oxidación de compuestos orgánicos volátiles hasta $\mathrm{CO}_{2}$. Por otra parte, es bien conocido la potenciación que muchas fases activas sufren al ser soportadas sobre un óxido cerámico como el $\mathrm{TiO}_{2}$ (11). Basándose en estos precedentes y previos trabajos realizados en el grupo de investigación sobre sistemas de óxidos mixtos de metales trivalentes ( $\mathrm{Al}, \mathrm{Cr}, \mathrm{Fe}$ y $\mathrm{Ga})(12,13,14)$, en este trabajo se presentan los resultados obtenidos en la caracterización de óxidos mixtos de Mn y Ti obtenidos por un método de impregnación húmeda, resaltando las propiedades catalíticas en la reacción de oxidación de isopropanol y el estudio de intermedios de reacción con espectroscopía FT-IR y análisis cromatográfico de gas-masas. 


\section{PROCEDIMIENTO EXPERIMENTAL}

\subsection{Preparación de las muestras}

Los óxidos mixtos de Mn y Ti se prepararon por un método de impregnación húmeda, partiendo de disoluciones acuosas de la sal precursora, $\mathrm{Mn}\left(\mathrm{CH}_{3} \mathrm{COO}\right)_{2} \cdot 2 \mathrm{H}_{2} \mathrm{O}$, conteniendo las cantidades requeridas de $\mathrm{Mn}$ para obtener los óxidos mixtos denotados como $\mathrm{XMn}_{\mathrm{MiO}}$ ( $\operatorname{con} \mathrm{X}=0.5,1.0$, 1.5 y 2.0, fracciones de la monocapa teórica) y añadiendo posteriormente el soporte $\mathrm{TiO}_{2}$ anatasa (Tioxide, $129 \mathrm{~m}^{2} / \mathrm{g}$ ). Posteriormente, los materiales se agitaron a $363 \mathrm{~K}$ hasta evaporar totalmente el agua y se calcinaron a $773 \mathrm{~K}$ durante $3 \mathrm{~h}$. Para calcular la cantidad de $\mathrm{MnO}_{\mathrm{x}}$ necesario para cubrir con una monocapa la superficie del soporte, se tuvo en cuenta el descenso en la superficie específica que se produce a la temperatura de calcinación de $773 \mathrm{~K}$ (descendió a $69 \mathrm{~m}^{2} / \mathrm{g}$ ) y las dimensiones de la celda unidad de la fase $\alpha-\mathrm{Mn}_{2} \mathrm{O}_{3}$-bixbyita $(15,16)$.

\subsection{Técnicas experimentales}

Los difractogramas de rayos $X$ se registraron en un difractómetro Philips PW 1710 (radiación $\mathrm{Cu} \mathrm{K \alpha}$, filtro Ni; $45 \mathrm{kV}, 35 \mathrm{~mA}$ ).

Las superficies específicas se midieron en un aparato volumétrico convencional (diseñado y construido en la Universidad de Salamanca) mediante la adsorción de nitrógeno a $77 \mathrm{~K}$.

Los análisis térmicos (DTA-TG) se realizaron, en atmósfera de aire, con una termobalanza Setaram TGA 92-12, desde temperatura ambiente hasta $1273 \mathrm{~K}$ a la velocidad de calentamiento de $10 \mathrm{~K} / \mathrm{min}$.

Los espectros FT-IR se registraron con un espectrómetro Nicolet Magna 750 con transformada de Fourier en la región 4000-50 $\mathrm{cm}^{-1}$. Los espectros FT-IR después de la adsorción de isopropanol sobre discos prensados de las muestras previamente tratadas se registraron después de evacuación y calentamiento a diferentes temperaturas.

Los espectros de reflectancia difusa visible-ultravioleta se realizaron en un espectrofotómetro JASCO V-570 en el intervalo $200-2500 \mathrm{~nm}$ utilizando pastillas prensadas de las muestras y un polímero como referencia.

Los espectros FT-Raman se registraron en un instrumento Brucker RFS100, con un láser Nd-YAG (1064 nm), utilizando $30 \mathrm{~mW}$ de potencia de láser, 2000 scans y $4 \mathrm{~cm}^{-1}$ de resolución.

Las pruebas catalíticas se realizaron a presión atmosférica en un reactor tubular de cuarzo en flujo continuo. El flujo total fue de $300 \mathrm{ml} / \mathrm{min}$ y la composición de isopropanol en la alimentación fue del 2\%. Las condiciones de preparación del catalizador y la metodología experimental se han descrito previamente (8). Estos catalizadores presentaron una gran estabilidad después de tres pruebas catalíticas sin alterar significativamente sus propiedades: superficie específica, difractograma $\mathrm{RX}$, etc...

Los reactivos y los productos de reacción se analizaron usando dos cromatógrafos de gases en línea (HP 5890), trabajando en diferentes condiciones para obtener la mejor resolución de las especies orgánicas e inorgánicas. El análisis de los productos mediante espectrometría de gasmasas (MS-GC) se realizó utilizando un aparato HP 1800D GCD Plus.
TAbla I. ParÁmetros de Celda, TAMAÑo de CRISTAL Y SUPERFICIE ESPECÍFICA DE LAS MUESTRAS CALCINADAS A $773 \mathrm{~K}$.

\begin{tabular}{|c|c|c|c|c|c|c|c|}
\hline Muestras & Fase & $\mathbf{D}(\AA)$ & a $(\AA)$ & b $(\AA)$ & c $(\mathbf{A})$ & $\mathbf{V}\left(\AA^{3}\right)$ & $\mathbf{S}\left(\mathrm{m}^{2} / \mathrm{g}\right)$ \\
\hline \multirow{2}{*}{$\mathrm{TiO}_{2}$} & A & 101 & $3.782(4)$ & $3.782(4)$ & $9.525(12)$ & 136.3 & \\
\hline & B & 136 & $5.452(11)$ & $9.207(40)$ & $5.147(63)$ & 258.4 & 69 \\
\hline \multirow{2}{*}{$0.5 \mathrm{Mn}-\mathrm{TiO}_{2}$} & A & 82 & $3.787(6)$ & $3.787(6)$ & $9.524(30)$ & 136.6 & \multirow{2}{*}{72} \\
\hline & B & 113 & $5.462(11)$ & $9.159(41)$ & $5.160(12)$ & 258.1 & \\
\hline \multirow{2}{*}{$1.0 \mathrm{Mn}-\mathrm{TiO}_{2}$} & A & 85 & $3.782(6)$ & $3.782(6)$ & $9.494(23)$ & 135.8 & \multirow{2}{*}{79} \\
\hline & B & 107 & $5.444(10)$ & $9.192(68)$ & $5.150(22)$ & 257.7 & \\
\hline \multirow{2}{*}{$1.5 \mathrm{Mn}-\mathrm{TiO}_{2}$} & A & 87 & $3.784(2)$ & $3.784(2)$ & $9.472(11)$ & 135.6 & \multirow{2}{*}{82} \\
\hline & B & 102 & $5.435(3)$ & $9.131(2)$ & $5.134(4)$ & 254.8 & \\
\hline \multirow{2}{*}{$2.0 \mathrm{Mn}-\mathrm{TiO}_{2}$} & A & 85 & $3.772(7)$ & $3.772(7)$ & $9.467(28)$ & 134.7 & \multirow{2}{*}{85} \\
\hline & B & 101 & $5.419(13)$ & $9.167(25)$ & $5.128(9)$ & 254.7 & \\
\hline
\end{tabular}

$\mathrm{A}=$ anatasa $; \mathrm{B}=$ brookita

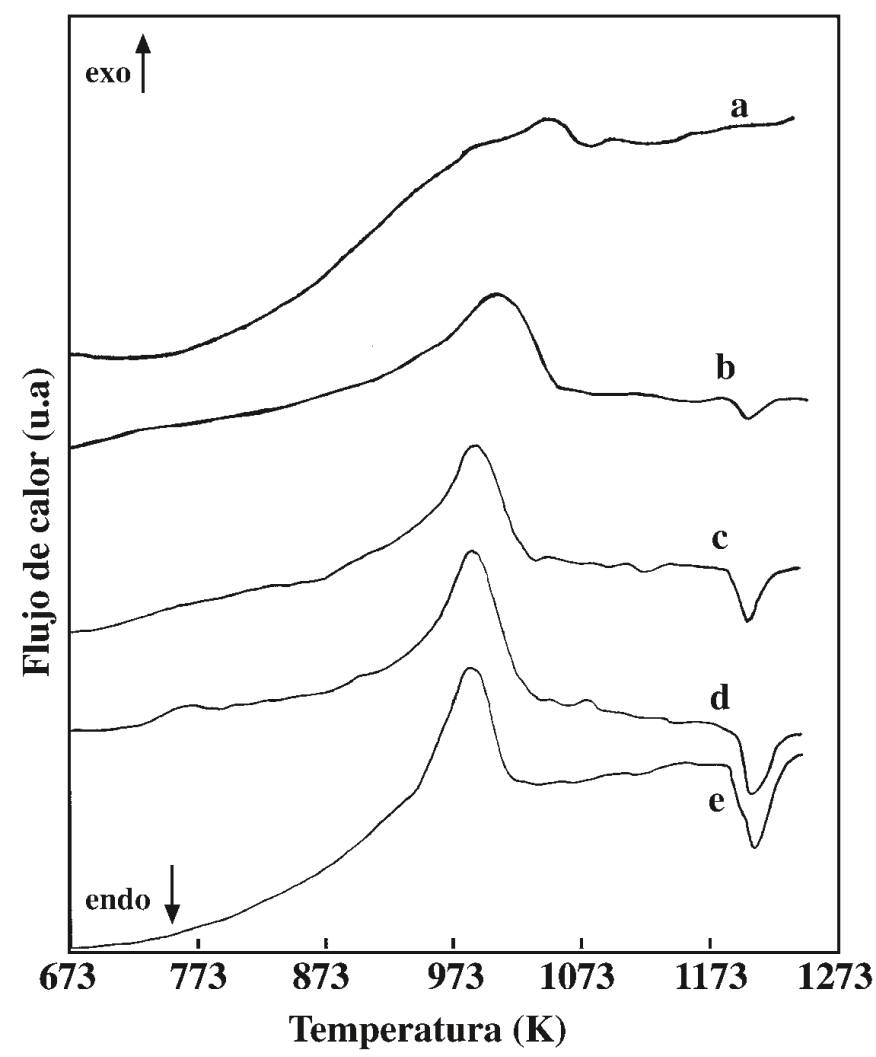

Fig. 1. Análisis térmico diferencial de las muestras calcinadas a $773 \mathrm{~K}$. a) $\mathrm{TiO}_{2}$, b) $0.5 \mathrm{Mn}-\mathrm{TiO}_{2}$, c) $1.0 \mathrm{Mn}-\mathrm{TiO}_{2}$, d) $1.5 \mathrm{Mn}-\mathrm{TiO}_{2}$, e) $2.0 \mathrm{Mn}-\mathrm{TiO}_{2}$. 


\section{RESULTADOS Y DISCUSIÓN}

\subsection{Caracterización de las muestras}

\subsubsection{EVOLUCIÓN DE LAS SUPERFICIES ESPECÍFICAS}

Los valores de las superficies específicas medidas muestran que la impregnación y calcinación a $773 \mathrm{~K}$ producen un descenso considerable del área del soporte (Tabla I), la cual presenta un valor inicial de $129 \mathrm{~m}^{2} / \mathrm{g}$. Sin embargo, esta disminución es más baja que la que experimenta el soporte $\mathrm{TiO}_{2}$. Además este efecto es mucho más bajo a mayores cargas de Mn, sugiriendo que la impregnación de Mn provoca una inhibición de la sinterización del $\mathrm{TiO}_{2}$ por simple calcinación a la misma temperatura.

\subsubsection{Difracción de RX y estabilidad térmica}

En la Tabla I se muestran las fases que componen los materiales, el tamaño de cristal, los parámetros y el volumen de celda obtenidos de los difractogramas de rayos X. Todas las muestras están constituidas por dos fases identificadas, anatasa (ICDD file $n^{\circ} 21-1272$ ) y brookita (ICDD file $\left.n^{\circ} 29-1360\right)$. Los picos de difracción son anchos y sus intensidades decrecen ligeramente con el aumento del contenido de $\mathrm{Mn}$, indicando materiales con un bajo grado de cristalinidad. Por otra parte los tamaños de cristal calculados según la fórmula de Scherrer (17), tienden a disminuir con el aumento del contenido de Mn en buen acuerdo con la inhibición de la sinterización discutida en el apartado anterior. Por otra parte, los parámetros de celda disminuyen ligeramente y aunque estar dentro del error de medida, existen estudios previos de la solubilidad existente entre las fases $\mathrm{MnO}_{2}$ y $\mathrm{TiO}_{2}$ (rutilo) (18) que implicaría que a esta temperatura existe cierta difusión del $\mathrm{Mn}$ hacia el interior de la estructura del $\mathrm{TiO}_{2}$. Estos datos son consecuentes con la disminución del tamaño del cristal y la pérdida de área superficial.

Desde el punto de vista de la estabilidad térmica, los materiales presentan una superficie específica media-alta a la temperatura de $773 \mathrm{~K}$ en comparación con materiales utilizados en oxidación catalítica como los óxidos de manganeso $\mathrm{Mn}_{3} \mathrm{O}_{4}$ y $\mathrm{Mn}_{2} \mathrm{O}_{3}\left(12-20 \mathrm{~m}^{2} / \mathrm{g}\right)(8)$ y de acuerdo con los análisis térmicos no presentados explícitamente, no se observan pérdidas de peso. Se deduce que la fase del óxido de manganeso no presenta fenómenos de oxidación como sucedía en estudios previos de sistemas mixtos de Mn-Ti obtenidos por coprecipitación (19). El análisis térmico diferencial (figura 1) presenta un pico exotérmico amplio asociado a la sinterización de la fase anatasa seguido de otro más agudo relacionado con la transición de fase anatasarutilo del $\mathrm{TiO}_{2}$ (20). La posición de los mismos varía hacia mayores y menores temperaturas, respectivamente, con el aumento del contenido de Mn. Esto está indicando que se produce una inhibición del proceso de sinterización de la fase anatasa y se favorece la transición de fase anatasa-rutilo, en buen acuerdo con los datos anteriores. También se observa un pico endotérmico relacionado con la formación de la fase $\mathrm{MnTiO}_{3}$ que aumenta en intensidad con el contenido de Mn en las muestras.

\subsubsection{CARACTERIZACIÓN ESPECTROSCÓPICA}

\subsubsection{Estudios FT-IR y FT-Raman}

Los espectros FT-Raman de las muestras calcinadas a $773 \mathrm{~K}$ se muestran en la figura 2. El espectro correspondiente al $\mathrm{TiO}_{2}$ (figura 2,a) presenta las bandas típicas del $\mathrm{TiO}_{2}$ anatasa a 645,

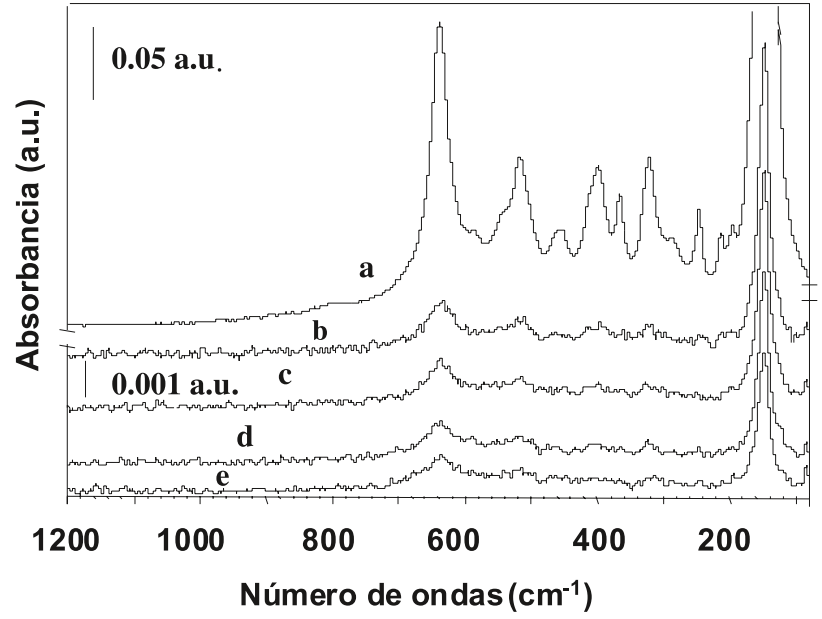

Fig. 2. Espectros FT-Raman de las muestras calcinadas a $773 \mathrm{~K}$. a) $\mathrm{TiO}_{2}$, b) $0.5 \mathrm{Mn}-\mathrm{TiO}_{2}$, c) $1.0 \mathrm{Mn}-\mathrm{TiO}_{2}$, d) $1.5 \mathrm{Mn}-\mathrm{TiO}_{2}$, e) $2.0 \mathrm{Mn}-\mathrm{TiO}_{2}$.

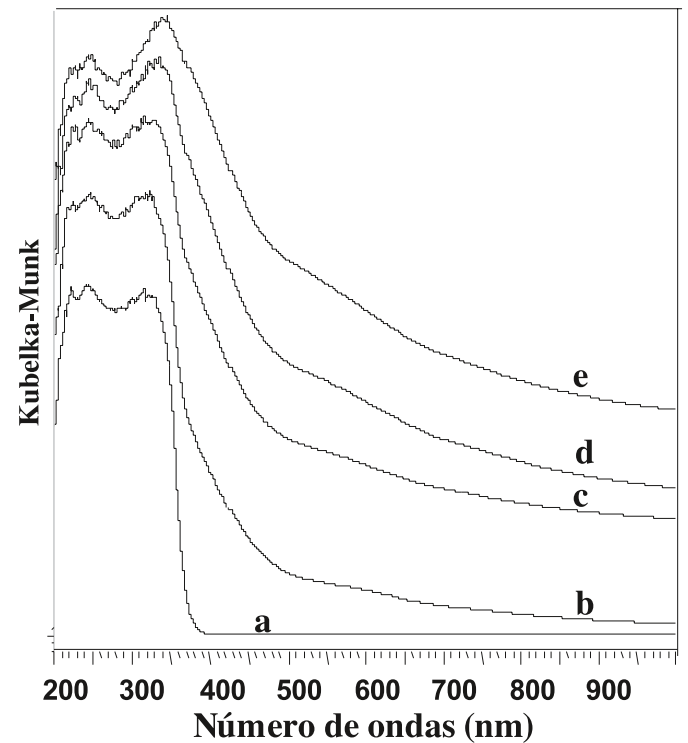

Fig. 3. Espectros UV-Vis de las muestras calcinadas a $773 \mathrm{~K}$. a) $\mathrm{TiO}_{2}$, b) $0.5 \mathrm{Mn}-\mathrm{TiO}_{2}$, c) $1.0 \mathrm{Mn}-\mathrm{TiO}_{2}$, d) $1.5 \mathrm{Mn}-\mathrm{TiO}_{2}$, e) $2.0 \mathrm{Mn}-\mathrm{TiO}_{2}$.

522, 390, 198 y $144 \mathrm{~cm}^{-1}$ además de aquellas de la fase brookita a $592,543,465,365,312,290$ y $225 \mathrm{~cm}^{-1}$, en buen acuerdo con los aportados previamente $(21,22,23)$. Cuando se añade $\mathrm{Mn}$, se observa un descenso muy acusado del efecto Raman que se ha atribuido al aumento de la absorción en la superficie $(21,24)$ y estaría indicando un buen recubrimiento de la superficie del $\mathrm{TiO}_{2}$ por el óxido de manganeso. Por otra parte, los espectros FT-IR debido a la baja cristalinidad de las muestras observada en difracción de rayos $X$ no muestran diferencias significativas. Con estas técnicas tampoco se encuentran evidencias de la presencia de fases de óxidos de manganeso.

\subsubsection{Espectroscopía Vis-UV}

Los espectros electrónicos del $\mathrm{TiO}_{2}$ y de las diferentes muestras impregnadas con $\mathrm{Mn}$ se comparan en la figura 3. Para el soporte $\mathrm{TiO}_{2}$ (figura 3,a), el espectro se caracteriza por una banda de adsorción divida en dos máximos a 245 y $315 \mathrm{~nm}$ con un límite de adsorción bien definido a 390 nm. Estas caracte- 
rísticas están de acuerdo con los espectros típicos del $\mathrm{TiO}_{2}$ anatasa, aportados previamente $(21,25)$ y se relacionan con la transición de transferencia de carga $\mathrm{O}^{2-} \rightarrow \mathrm{Ti}^{4+}$. Cuando se añade $\mathrm{Mn}$, aparecen nuevas bandas en la zona de 400-500 nm, próxima a $585 \mathrm{~nm}, \mathrm{y}$, a más altos cargas de $\mathrm{Mn}$, una tercera banda débil próxima a $740 \mathrm{~nm}$ (figura 3,b,c). De acuerdo con estudios previos relacionados con óxidos de Mn-Ti coprecipitados (19) y disoluciones sólidas de $\mathrm{MnO}_{2}-\mathrm{TiO}_{2}$ tipo rutilo $(18,26)$, la banda de absorción justo por encima del límite de absorción de la anatasa $(400-450 \mathrm{~nm})$ puede relacionarse con $\mathrm{Mn}^{4+}$ que ha entrado en la estructura del $\mathrm{TiO}_{2}$ en buen acuerdo con lo datos de difracción de rayos X. Las componentes a 585 y $740 \mathrm{~nm}$ son debidas razonablemente a las transiciones $\mathrm{d} \rightarrow \mathrm{d}$ del campo de cristal de $\mathrm{Mn}^{3+}$ octaédrico sobre la superficie del $\mathrm{TiO}_{2}(27,28)$.

Estos óxidos de $\mathrm{Mn}$-Ti preparados por impregnación no presentan absorción sobre $800 \mathrm{~nm}$, en contraste con aquellos preparados por coprecipitación (19), indicando que no hay segregación de óxidos de Mn del "bulk".

\subsection{Pruebas catalíticas}

\subsubsection{ESTUDIO DE LA OXIDACIÓN DE ISOPROPANOL EN EL REACTOR DE FLUJO}

Las conversiones de 2-propanol sobre las distintas muestras en la reacción oxidación a diferentes temperaturas se comparan en la figura 4. La conversión de isopropanol comienza a observarse a $375 \mathrm{~K}$ y es total próxima a $525 \mathrm{~K}$ sobre el óxido de manganeso puro, $\mathrm{Mn}_{3} \mathrm{O}_{4}$. A esta temperatura, sobre el mejor catalizador de $\mathrm{Mn}-\mathrm{TiO}_{2}$ la conversión de isopropanol es solamente el $50 \%$ en las mismas condiciones. Para todos los catalizadores de $\mathrm{Mn}-\mathrm{TiO}_{2}$ la conversión se aproxima al $100 \%$ por encima de $600 \mathrm{~K}$. El catalizador más activo entre los óxidos de $\mathrm{Mn}$-Ti es aparentemente $1.5 \mathrm{Mn}-\mathrm{TiO}_{2}$, sugiriendo que hay una composición óptima por encima de la composición teórica de un catalizador tipo monocapa.

Sobre el $\mathrm{Mn}_{3} \mathrm{O}_{4}$ (no mostrado explícitamente), la oxidación de isopropanol produce casi exclusivamente acetona hasta 510 $\mathrm{K}$ (selectividad $>85 \%$ ) con pequeñas cantidades de acetaldehído y trazas de ácido acético y propeno como principales productos secundarios. A temperaturas más altas la selectividad a acetona cae rápidamente a cero, mientras la selectividad a $\mathrm{CO}_{2}$ crece por encima del $95 \%$ a $535 \mathrm{~K}$. Las selectividades observadas para los catalizadores de $\mathrm{Mn}-\mathrm{TiO}_{2}$ difieren bastante en relación con la carga de Mn. En todos los casos, las selectividades a acetona decrecen progresivamente desde $100 \%$ a cero con el incremento de la conversión de propeno (figura 5). Este decrecimiento de selectividad es más rápido cuánto más bajo es el contenido de $\mathrm{Mn}$, porque más propeno se produce en el intervalo 500-600 K (hasta un 30\% de selectividad para la muestra $0.5 \mathrm{Mn}-\mathrm{TiO}_{2}$ ) (figura 6). Mayores incrementos de la temperatura de reacción causan la oxidación total a $\mathrm{CO}_{2}(98 \%$ de selectividad, por encima de $625 \mathrm{~K}$ ) (figura 5, 6).

De acuerdo con estudios previos (29), la conversión de isopropanol sobre $\mathrm{TiO}_{2}$ es todavía muy baja a $473 \mathrm{~K}(<3 \%)$ y es completa sobre $550 \mathrm{~K}$ donde el producto predominante es propeno. Por lo tanto, cuando decrece la carga de $\mathrm{Mn}$, parte de la superficie de $\mathrm{TiO}_{2}$ permanece expuesta al reactivo y se transforma principalmente a propeno. Por el contrario, los centros con óxido de manganeso favorecen la oxidación a acetona. De este modo, tienen lugar dos reacciones paralelas a temperaturas medias dependiendo directamente de las dos clases de sitios expuestos, bien óxido de Mn u óxido de Ti.

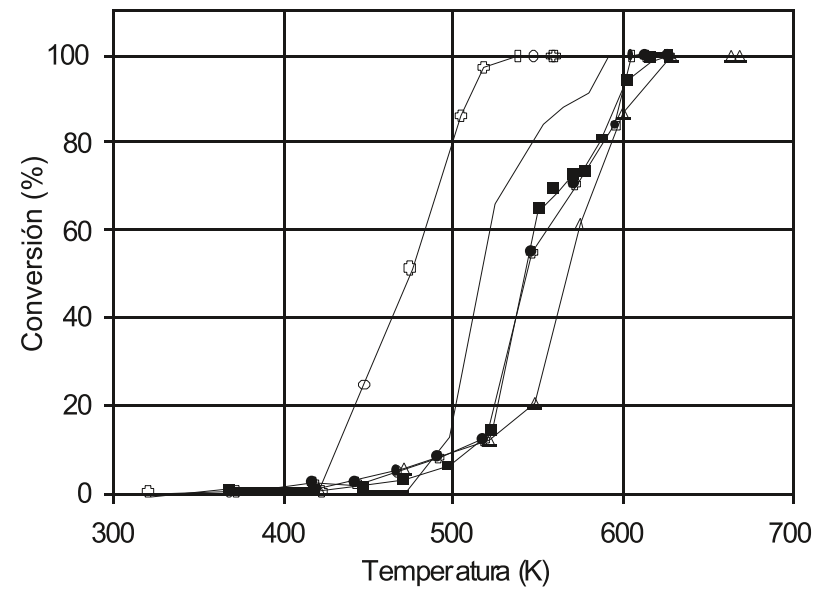

Fig. 4. Comparación de las conversiones de isopropanol en función de la temperatura de reacción para las muestras $\mathrm{Mn}_{3} \mathrm{O}_{4}(\mathrm{O}), 2.0 \mathrm{Mn}-\mathrm{TiO}_{2}($ $\square), 1.5 \mathrm{Mn}-\mathrm{TiO}_{2}(\bullet), 1.0 \mathrm{Mn}-\mathrm{TiO}_{2}(\boldsymbol{\bullet}), 0.5 \mathrm{Mn}-\mathrm{TiO}_{2}(\Delta)$.

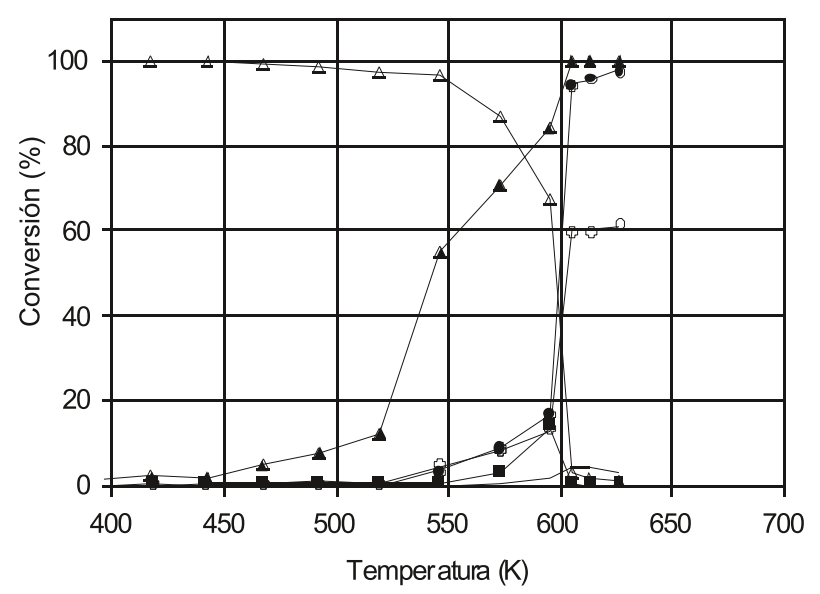

Fig. 5. Conversión de isopropanol y selectividades a los principales productos detectados sobre $2.0 \mathrm{Mn}-\mathrm{TiO}_{2} . \mathrm{O}_{2}(\mathbf{O}), \mathrm{CO}_{2}(\bullet), \mathrm{CO}(\square)$, $\mathrm{C}_{3} \mathrm{H}_{6}(\mathbf{\square}), \mathrm{CH}_{3} \mathrm{COCH}_{3}(\Delta), \mathrm{CH}_{3} \mathrm{CHOHCH}_{3}(5)$.

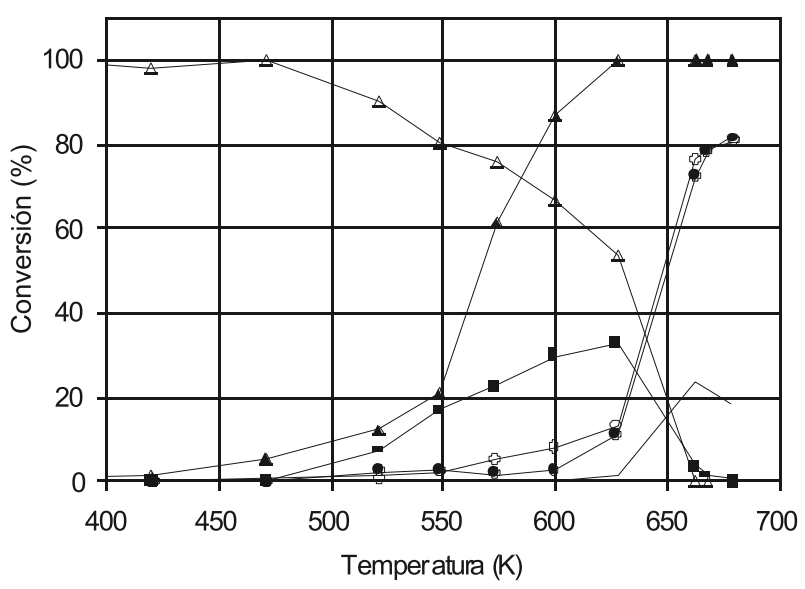

Fig. 6. Conversión de isopropanol y selectividades a los principales productos detectados sobre $0.5 \mathrm{Mn}-\mathrm{TiO}_{2} . \mathrm{O}_{2}(\mathbf{O}), \mathrm{CO}_{2}(\bullet), \mathrm{CO}(\square)$, $\mathrm{C}_{3} \mathrm{H}_{6}(\mathbf{\square}), \mathrm{CH}_{3} \mathrm{COCH}_{3}(\Delta), \mathrm{CH}_{3} \mathrm{CHOHCH}_{3}(5)$. 


\subsubsection{ESTUDIOS FT-IR DE LA ADSORCIÓN Y OXIDACIÓN DE ISOPROPANOL}

Los espectros FT-IR de las especies formadas en la superficie después de la interacción de isopropanol sobre la muestra $2.0 \mathrm{Mn}-\mathrm{TiO}_{2}$ se comparan en la figura 7. A temperatura ambiente, la adsorción de isopropanol da lugar a un espectro constituido por bandas situadas a 1466, 1380 (doblete), 1332, 1300 (ancha y débil), 1165 y $1127 \mathrm{~cm}^{-1}$. La banda de absorción ancha a $1300 \mathrm{~cm}^{-1}$ desaparece rápidamente después de calentar a 373 K (figura 7,b). De acuerdo con los datos de la literatura $(30,31,32)$, las bandas resistentes al calentamiento a $373 \mathrm{~K}$ se relacionan con las deformaciones asimétrica y simétrica del $\mathrm{CH}_{3}\left(1466,1380 \mathrm{~cm}^{-1}\right)$, deformación $\mathrm{CH}\left(1332 \mathrm{~cm}^{-1}\right)$, y tensiones C-C y C-O (1165 y $\left.1127 \mathrm{~cm}^{-1}\right)$, respectivamente, de las especies isopropóxido. La banda amplia próxima a $1300 \mathrm{~cm}^{-1}$ es debida a la deformación C-O-H de isopropanol adsorbido coordinativamente, que desorbe fácilmente. Las bandas de las especies isopropóxido van disminuyendo progresivamente la intensidad y desaparecen completamente a $573 \mathrm{~K}$ (figura 7,f). En paralelo, se encuentran nuevas bandas a $1682\left(v_{\mathrm{C}=\mathrm{O}}\right)$ y 1244 $\left(v_{\text {asy C-C-C }}\right) \mathrm{cm}^{-1}$ en el invertavalo $473-523 \mathrm{~K}$, asociadas con acetona adsorbida (31). Además, dos bandas de absorción próximas a 1585 y $1465 \mathrm{~cm}^{-1}$ aumentan la intensidad al incrementar la temperatura relacionadas con especies acetato (31), mientras que una absorción persiste próxima a $1380 \mathrm{~cm}^{-1}$, probablemente debidas a especies formiato. Después del calentamiento a $523 \mathrm{~K}$ (Fig. 6,e) las bandas de carboxilatos (principalmente acetatos) son todavía evidentes a 1560 y $1455 \mathrm{~cm}^{-1}$. Los espectros de la zona de tensión de los $\mathrm{CH}$ están de acuerdo con el cuadro descrito anteriormente.

En las mismas condiciones, se registraron los espectros de las especies de la fase gas (figura 8). Las bandas debidas al vapor de isopropanol disminuyen la intensidad progresivamente después del calentamiento, mientras aquellas debidas a vapor de acetona $\left(1738 \mathrm{~cm}^{-1}\right.$, tensión $\left.\mathrm{C}=\mathrm{O}\right)$ se observan comenzando desde $440 \mathrm{~K}$, crecen hasta $523 \mathrm{~K}$ y después disminuyen en intensidad progresivamente. El propeno gaseoso $\left(912 \mathrm{~cm}^{-1}\right.$, deformación $\mathrm{CH}_{2}$ ) comienza a detectarse desde $473 \mathrm{~K}$ y presenta una evolución análoga a la de la acetona, mientras que las bandas debidas a $\mathrm{CO}_{2}\left(2340\right.$ y $\left.667 \mathrm{~cm}^{-1}\right)$ llegan a ser predominantes por encima de $673 \mathrm{~K}$. La comparación de los espectros de la superficie y de la fase gas sugiere que los grupos isopropóxido son precursores del propeno en fase gas y acetona en fase gas y superficie, mientras $\mathrm{CO}_{2}$ es probablemente el producto final de oxidación de acetona a través de especies acetato y formiato. Los espectros obtenidos están de acuerdo con el mecanismo propuesto anteriormente para el $\mathrm{Mn}_{3} \mathrm{O}_{4}$ (33). Los espectros obtenidos con el resto de las muestras $\mathrm{Mn}-\mathrm{TiO}_{2}$ se diferencian de los discutidos aquí en la intensidad relativa de las bandas y los intervalos de temperatura de las especies de la superficie.

\subsubsection{ANÁLISIS CROMATOGRÁFICO DE GAS-MASAS DE LOS PRODUCTOS SECUNDARIOS DE LA OXIDA- CIÓN DE ISOPROPANOL}

Como se dijo anteriormente, el análisis de cromatografía de gases de los productos a la salida del reactor después de la oxidación de isopropanol mostró la presencia de isopropanol, oxígeno, acetona, y trazas de $\mathrm{CO}$, acetaldehído como principales productos. Sin embargo, en los cromatogramas existían pequeños picos difíciles de identificar por su pequeña intensidad. Para obtener más información sobre el mecanismo de reacción,

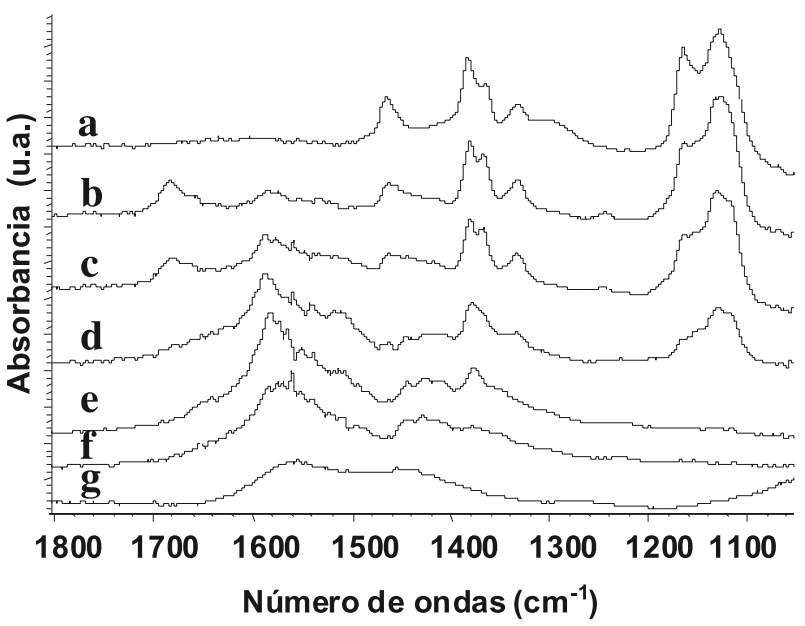

Fig. 7. Espectros FT-IR de las especies obtenidas después de la interacción del isopropanol sobre la muestra $2.00 \mathrm{Mn}-\mathrm{TiO}_{2}$, tras la evacuación y calentamiento a: a) $293 \mathrm{~K}$, b) $373 \mathrm{~K}, \mathrm{c}) 423 \mathrm{~K}$, d) $473 \mathrm{~K}$, e) $523 \mathrm{~K}$, f) $573 \mathrm{~K} \mathrm{y} \mathrm{g)} 673 \mathrm{~K}$.

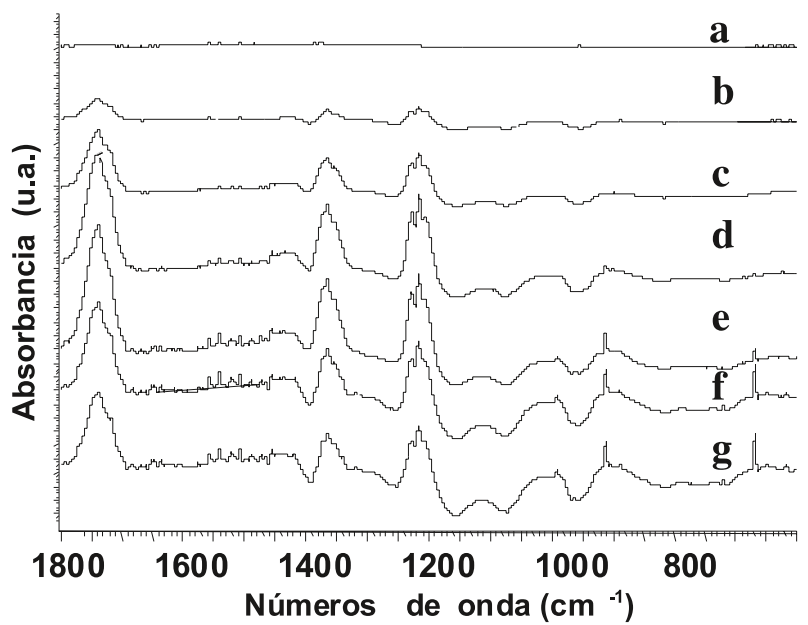

Fig. 8. Espectros FT-IR de las especies obtenidas después de la interacción del isopropanol sobre la muestra $2.00 \mathrm{Mn}-\mathrm{TiO}_{2}$ a las temperaturas: a) $373 \mathrm{~K}$, b) $443 \mathrm{~K}$, c) $473 \mathrm{~K}$, d) $523 \mathrm{~K}$, e) $573 \mathrm{~K}$, f) $623 \mathrm{~K}$ y g) $673 \mathrm{~K}$. (el espectro del isopropanol gas se ha sustraido).

se repitieron algunos experimentos con el análisis cromatográfico de gas-masas. Los tres picos a tiempos de retención de 11.5, 12.4 y 15.6 min en una columna capilar VOC-HP, observados para la muestra $2.0 \mathrm{Mn}-\mathrm{TiO}_{2}$ a $575 \mathrm{~K}$ a baja conversión, se muestran en la figura 9. Los espectros de masas se pueden observar también en la figura 9. Estos picos se identifican automáticamente como formiato de isopropilo (tiempo de retención de 11.5 min) éter isopropílico (12.4 $\mathrm{min}$ ) y acetato isopropílico (15.6 min). Estas especies desaparecen a conversiones más altas del reactivo. La detección de estas especies se puede relacionar con las observaciones anteriores de la espectroscopía FT-IR de los grupos formiatos, isopropóxidos y acetatos en la superficie del catalizador. Parece probable que el isopropanol cuanto está presente en grandes cantidades, arrastra estas especies de la superficie y las atrapa como ésteres y éteres. De este modo, estos datos se pueden tomar como una evidencia del papel de los grupos isopropóxido, acetatos y formiatos en el mecanismo de oxidación de isopropanol (ver la figura 10). 


\section{CONCLUSIONES}

Las principales conclusiones encontradas en este trabajo se pueden resumir de la siguiente manera:

Los óxidos de Mn-Ti preparados por impregnación de un soporte $\mathrm{TiO}_{2}$ anatasa conteniendo brookita están formados por óxidos de manganeso bien dispersados por la superficie y también por $\mathrm{MnO}_{2}$ que ha entrado parcialmente en la estructura del $\mathrm{TiO}_{2}$. No se detectan trazas de óxidos de manganeso como fase aislada incluso a altos contenidos de $\mathrm{Mn}$.

El óxido de Mn impregnado parece inhibir la sinterización de la fase anatasa, mientras que favorece la transición de fase anatasa-rutilo.

Los espectros electrónicos sugieren que la existencia de $\mathrm{Mn}^{4+}$ en el interior de la estructura y el predominio de $\mathrm{Mn}^{3+}$ en la superficie.

Los óxidos de $\mathrm{Mn}$ soportados sobre $\mathrm{TiO}_{2}$ son menos activos que los óxidos de Mn puros en la oxidación de isopropanol, y también menos selectivos a acetona a conversiones incompletas. No obstante, se aumenta la selectividad a propileno para bajas cargas de Mn.

Las muestras con alto contenido de Mn presentan superficies específica medio-altas (con un valor medio de $80 \mathrm{~m}^{2} / \mathrm{g}$ ) muy estables en el intervalo $600-900 \mathrm{~K}$, produciendo en este intervalo solamente $\mathrm{CO}_{2}$.

Los estudios FT-IR y análisis GC-MS de algunos intermedios de reacción permiten confirmar que la oxidación completa de isopropanol procede a través del mecanismo de superficie previamente propuesto (figura 10) isopropóxido/acetato/formiato.

$\mathrm{La}$ actividad catalítica de las muestras $\mathrm{Mn}-\mathrm{TiO}_{2}$ respecto al $\mathrm{Mn}_{3} \mathrm{O}_{4}$ (medida por unidad de área) se atribuye a la disolución de los sitios activos $\mathrm{Mn}^{\mathrm{n}+}$ en el $\mathrm{TiO}_{2}$.

\section{AGRADECIMIENTOS}

Los autores agradecen la financiación de este trabajo a la OTAN (CRG-960316) y a la Junta de Castilla y León (SA37/98). JMGA agradece, en especial, al MEC la concesión de una beca FPI, postdoctoral.

\section{BIBLIOGRAFÍA}

1. A. Wollner, F. Lange, H. Schmelz, H. Knozinger. “Characterization of Mixed Copper-Manganese Oxides Supported on Titania Catalysts for Selective Oxidation of Ammonia". Appl. Catal., A: General 94 181-203 (1993).

2. W.S. Kiljlstra, E.K. Poels, A.Bliek, B.M. Weckhuysen, R.A. Schoonheydt. "Characterization of $\mathrm{Al}_{2} \mathrm{O}_{3}$-supported Manganese Oxides by Electron Spin Resonance and Diffuse Reflactance Spectroscopy" J. Phys. Chem. B 101, 309316 (1997).

3. Y. Li, J.N. Armor. "Selective Catalytic Reduction of NO with Methane on Co-Ferrierites" J. Catal. 145 1-9(1994).

4. X. Zhang, A.B. Walters, M.A. Vannice. “Catalytic Reduction of $\mathrm{NO}$ by $\mathrm{CH}_{4}$ over Li-promoted MgO" J. Catal. 146 568-578 (1994).

5. Sulphur 187 (1986); B.G. Gore. J. Oil \& Gas, 23 (May), 61-65 (1994).

6. E. Astorino, G. Busca, G. Ramis, R.J. Willey. "FT-IR study of the interaction of magnesium ferrite with $\mathrm{SO}_{2}$ " Catal. Letters 23 353-360 (1994).

7. C. M. Gouveia Capelão,J. Urbano. “La catálisis en la Industria de Refinado de Petróleos" en Proceedings of III National Meeting on Basic and Applied Catalysis (Industrial and Enviromental)", Lisboa 1995.

8. M. Baldi, E. Finocchio, F. Milella, G. Busca. "Catalytic combustion of C3 hydrocarbons and oxygenates over $\mathrm{Mn}_{3} \mathrm{O}_{4}$ " Appl. Catal. B: Environ. 16 43-51 (1998).

9. J.E. Germain, R. Laugier. "Kinetics of Catalytic Oxidation of Aromatic Hydrocarbons Activity and Selectivity Spectra of Metallic Oxides in the Catalysis of Toluene Oxidation" Bull. Soc. Chim. France 541-548 (1972).

10. C. Lahousse, A. Bernier, A. Gaigneaux, P. Ruiz, P. Grange, B. Delmon, in R.K. Grasselli et al. (Eds.), 3rd World Congress on Oxidation Catalysis, Elsevier, Amsterdam p. 777 (1997).

11. B. Grzybowska-Swierkosz, F. Trifirò, J.C. Vedrine“Vanadia catalysts for

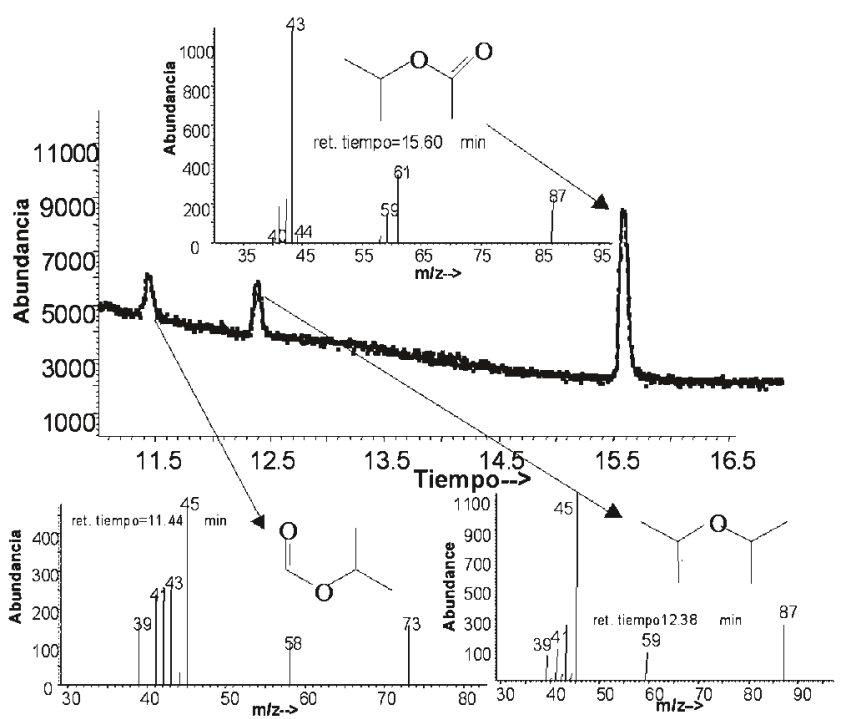

Fig. 9. Cromatograma gas-masas de los productos obtenidos de la oxidación de isopropanol sobre $2.00 \mathrm{Mn}-\mathrm{TiO}_{2}$ a $573 \mathrm{~K}$

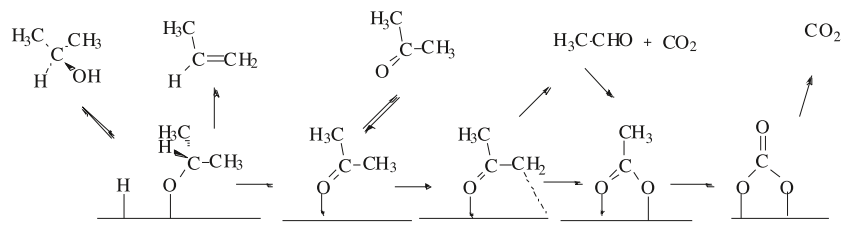

Fig. 10. Esquema del mecanismo de reacción en la superficie propuesto para los óxidos mixtos de $\mathrm{Mn}-\mathrm{TiO}_{2}$.

selective oxidation of hydrocarbons and their derivatives", eds., App. Catal. A: General 157 1-426 (1997).

12. V.S. Escribano, J.M. Gallardo, E. Finocchio, M. Daturi, G. Busca "Characterization of a- $(\mathrm{Fe}, \mathrm{Al})_{2} \mathrm{O}_{3}$ Solid Solution Powders" J. Mater. Chem. 5(11) 1943-1945 (1995).

13. J.M. Gallardo-Amores, M.C. Prieto, V.S. Escribano, C. Cristiani, M. Trombetta, G. Busca. "Characterization of coprecipitated aluminun-chromium mixed hydroxides and of the products of their calcination" J. Mater. Chem. 7(9) 1887-1893 (1997)

14. J.M. Gallardo-Amores, V. Sánchez-Escribano, G. Busca. “Characterization of Fe Ga mixed hydroxides powders" J. Mater. Chem. 9(5) 1161-1166 (1999).

15. M. Baldi, V. Sanchez Escribano, J.M. Gallardo-Amores, F. Milella, G. Busca. "Characterization of manganese and iron oxides as combustion catalysts for propane and propene" Appl. Catal. B: Environ. 17 L-175- L182 (1998).

16. B.G. Hyde, S. Andersson. "Inorganic Crystal Structures", 1st edn., Wiley Intersience, New York (1989).

17. A.R. West. "Solid State Chemistry and its Applications", 1st edn., New York (1989)

18. M. Valigi, C. Cimino. “The system $\mathrm{TiO}_{2}-\mathrm{MnO}_{\mathrm{x}}$ : A structural, thermogravimetric and magnetic study" J. Solid State Chem. 12 135-143 (1975).

19. F. Milella, J.M. Gallardo-Amores, M. Baldi, G. Busca. “A study of Mn-Ti oxide powders and their behaviour in propane oxidation catalysis" J. Mater. Chem. 8 2525-2531 (1998).

20. J.M. Gallardo, V.S. Escribano, G. Busca. “Anatase Crystal Growth and Phase Transformation to Rutile in High-area $\mathrm{TiO}_{2}, \mathrm{MoO}_{3}-\mathrm{TiO}_{2}$ and $\mathrm{TiO}_{2}$-supported Oxide Catalytic Systems" J. Mater. Chem. 5 1245-1249 (1995).

21. G. Busca, J.M. Gallardo, P. Piagio, G. Ramis, V. S. Escribano. “FT-Raman and FT-IR studies of titanias and metatitanate powders" J. Chem. Soc., Faraday Trans. 90 3181-3190 (1994).

22. T. Osaka, F. Uzumi, Y. Fujiki. "Raman spectrum of anatase, $\mathrm{TiO}_{2}$ " J. Raman Spectrosc. 7 321-324 (1978).

23. Lj. D. Arsov, C. Kormann, W. Plieth. “Electrochemical synthesis and in situ Raman spectroscopy of thin films of titanium dioxide" J. Raman Spectrosc. 22 573-575 (1991).

24. G.C. Bond, J. Perez Zurita, S. Flamerz, P.J. Gellings, H. Borch, J.G. Van Ommen, B.J. Kip. "Structure and reactivity of titania-supported oxides. Part 1: Vanadium Oxide on Titania in the sub- and super- Monolayer Regions" Appl. Catal. 22 361-378 (1986).

25. J.M. Gallardo, V.S. Escribano, G. Busca, V. Lorenzelli. "Solid State and 
Surface Chemistry of the CuO-TiO 2 (Anatase) Catalytic System" J. Mater. Chem. 4 965-971 (1994).

26. D. Cordischi, M. Valigi, D. Gazzoli, V. Indovina. "ESR and Reflectance Spectra of Manganese in Polycrystilline $\mathrm{TiO}_{2}$ (Rutile)" J. Solid State Chem. 15 82-88 (1975)

27. J.R. Akrige, J.H. Kennedy. "Absorption and Emission Spectroscopy and Magnetic Susceptibility of Sodium b-alumina doped with Mn, Co and Ni" J. Solid State Chem. 29 63-72 (1979)

28. M. Lenglet, M. Bizi, C.K. Jorgensen. “Influence de la Distribution Cationique et de la Nature des Interactions Magnetiques sur l'intensite des Transitions de Paires de l'ion $\mathrm{Fe}^{3+}$ dans quelques Spinelles Mixtes" J. Solid State Chem. 86 82-87 (1990)

29. G. Busca, V. Sanchez Escribano, P. Forzatti, L. Lietti, G. Ramis in “New Developments in Selective Oxidation", V. Cortes Corberan and S. Vic Bellon eds., Elsevier, Amsterdam, p. 777 (1994).
30. P.F. Rossi, G. Busca, V. Lorenzelli, O. Saur, J.C. Lavalley. “Microcalorimetric and FT-IR spectroscopic study of the adsorption of isopropyl alcohol and hexafluoro-isopropyl alcohol on titanium dioxide" Langmuir 3 52-58 (1987).

31. V. Sanchez Escribano, G. Busca, V. Lorenzelli. "FT-IR studies of the reactivity of vanadia-titania catalysts towards olefins. Part.III: n-butenes and isobutene" J. Phys. Chem. 95 5541-5545 (1991).

32. E. Finocchio, G. Busca, V. Lorenzelli, V. Sanchez Escribano. “FT-IR studies on the selective oxidation and combustion of light hydrocarbons at metal oxide surfaces. Propane and propene oxidation on Co3O4" J. Chem. Soc., Faraday Trans. 92 1587-1593 (1996)

33. M. Baldi, F. Milella, G. Ramis, V. Sánchez Escribano, G. Busca. “An FT-IR and flow reactor study of the selective catalytic oxy-dehydrogenation of C3 alcohols on Mn3O4" App. Catal. A: General 166 75-88 (1998). 